\title{
Association of microsatellite polymorphisms of the GPDSI locus with normal tension glaucoma in the Japanese population
}

Kayo Nakamura ${ }^{{ }^{*}}$, Masao Ota ${ }^{2 *}$ Akira Meguro', Naoko Nomura' Kenji Kashiwagi ${ }^{3}$, Fumihiko Mabuchi ${ }^{3}$ Hiroyuki lijima ${ }^{3}$, Kazuhide Kawase ${ }^{4}$

Tetsuya Yamamoto ${ }^{4}$, Makoto Nakamura ${ }^{5}$ Akira Negi ${ }^{5}$, Takeshi Sagara ${ }^{6}$

Teruo Nishida ${ }^{6}$, Masaru Inatani ${ }^{7}$ Hidenobu Tanihara ${ }^{7}$, Makoto Aihara ${ }^{8}$ Makoto Araie ${ }^{8}$, Takeo Fukuchi ${ }^{9}$ Haruki Abe9, Tomomi Higashide ${ }^{10}$ Kazuhisa Sugiyama ${ }^{10}$,Takashi Kanamoto"1 Yoshiaki Kiuchi'", Aiko Iwase ${ }^{12}$ Shigeaki Ohno ${ }^{13}$, Hidetoshi Inoko ${ }^{14}$ Nobuhisa Mizuki

'Department of Ophthalmology, Yokohama City University School of Medicine,

Yokohama, Kanagawa, Japan; ${ }^{2}$ Department of Legal Medicine, Shinshu University School of Medicine, Matsumoto, Nagano, Japan; ${ }^{3}$ Department of Ophthalmology, University of Yamanashi, Faculty of Medicine, Yamanashi, Japan; ${ }^{4}$ Department of Ophthalmology, Gifu University Graduate School of Medicine, Gifu, Japan; ${ }^{5}$ Department of Surgery, Division of Ophthalmology, Kobe University Graduate School of Medicine, Kobe, Hyogo, Japan;

${ }^{6}$ Department of Ophthalmology, Yamaguch University Graduate School of Medicine, Ube, Yamaguchi, Japan; 'Department of Ophthalmology and Visual Science, Graduate School of Medical Sciences, Kumamoto University, Kumamoto, Japan; ${ }^{8}$ Department of Ophthalmology, University of Tokyo School of Medicine, Tokyo, Japan; 'Division of Ophthalmology and Visual

Science, Graduated School of Medical and Dental Sciences, Niigata University, Niigata, Japan; ${ }^{\circ}$ Department of Ophthalmology and Visual Science, Kanazawa University Graduate School of Medical Science, Kanazawa, Ishikawa, Japan; "Department of Ophthalmology and Visual Science, Graduate School of Biomedical Sciences, Hiroshima University, Hiroshima, Japan; ${ }^{12}$ Department of Ophthalmology, Tajimi Municipal Hospital, Tajimi, Gifu, Japan;

${ }^{13}$ Department of Ophthalmology, Hokkaido University School of Medicine, Sapporo, Japan; ${ }^{14}$ Department of Genetic Information, Division of Molecular Life Science,

Tokai University School of Medicine,

Isehara, Kanagawa, Japan; *These authors contributed equally to this report.

Correspondence: Akira Meguro Department of Ophthalmology and Visual Science, Yokohama City University Graduate School of Medicine,

3-9 Fukuura, Kanazawa-ku, Yokohama,

Kanagawa 236-0004, Japan

Tel +8I 457872683

Fax $+8|4578| 9755$

Email akmeguro@yokohama-cu.ac.jp
Background: To investigate whether the GPDS1 locus, a potential causative locus of pigment-dispersion syndrome, is associated with normal-tension glaucoma (NTG) in Japanese patients.

Materials and methods: We used polymerase chain reaction amplification with sequencespecific primers to analyze 20 polymorphic microsatellite markers in and around the GPDS1 locus with an automated DNA analyzer and automated fragment detection by fluorescent-based technology. The DNA samples used for these analyses were obtained from ethnicity- and gendermatched patients, including 141 Japanese patients with NTG and 101 healthy controls. Patients exhibiting a comparatively early onset were selected as this suggests that genetic factors may show stronger involvement.

Results: One allele of D7S2462 exhibited a frequency that was significantly decreased in NTG cases compared to controls $(\mathrm{P}=0.0013, \mathrm{Pc}=0.019, \mathrm{OR}=0.48,95 \% \mathrm{CI}=0.30-0.75)$. Alleles at another six microsatellite loci were positively or negatively associated with NTG, but these associations did not retain statistical significance after Bonferroni correction $(\mathrm{P}<0.05, \mathrm{Pc}>0.05)$.

Conclusion: Our study showed a significant association between the GPDS1 locus and NTG, suggesting that there may be some genetic risk factor(s) in the development of NTG.

Keywords: microsatellite, normal tension glaucoma, glaucoma-related pigment dispersion syndrome, GPDS1, DPP6

\section{Background}

Glaucoma is the second most common cause of blindness worldwide. ${ }^{1}$ This condition has a high socioeconomic impact, affecting over 67 million people in the western world. ${ }^{2}$ The disease is characterized by a loss of visual field corresponding to the excavation of the optic disc. It is considered to be a multifactorial disorder that occurs because of the interaction of genetic and environmental factors. In addition to an association with intraocular pressure (IOP), the onset and progression of glaucoma is involved with several other risk factors, including ischemia, refraction, systemic illness, and genetic predisposition.

Primary open-angle glaucoma (POAG) is usually associated with elevated IOP. The pathogenesis of glaucomatous optic neuropathy is not completely understood, although elevated IOP is a generally accepted major risk factor for this condition. Normal-tension glaucoma (NTG) is an important subtype of POAG and accounts for approximately one-third of all POAG cases in Caucasians. ${ }^{3}$ Notably, the incidence of NTG is higher in Japan than in the western world, and it occurs in approximately $92 \%$ of all Japanese POAG patients, as reported in the Tajimi Study. ${ }^{4}$ The IOP of NTG patients consistently lies within the statistically normal range; thus, although IOP is suggested to play a role in the development of NTG, its involvement may be less in NTG than that in high-tension glaucoma (HTG). Other pathogenic factors such as vasospasm, 
ischemia, and vascular insufficiencies are indicated to be associated with the development of NTG. ${ }^{5-8}$

Pigment dispersion syndrome (PDS) and pigmentary glaucoma (PG) are characterized by a disruption of iris pigment epithelium and deposition of the dispersed pigment granules throughout the anterior segment. The classic diagnostic triad consists of corneal pigmentation (Krukenberg spindle); slit-like, radial, or mid-peripheral iris transillumination defects; and dense trabecular pigmentation. ${ }^{9} \mathrm{PDS} / \mathrm{PG}$ usually affects individuals under the age of 30 years. In the early stages of the disease, affected individuals may exhibit clinical evidence of dispersed pigment without an associated elevation of IOP or optic nerve degeneration. However, as the disease progresses, some patients develop increased IOP and degeneration of the optic nerve, causing permanent vision loss. Recent linkage studies have identified several genes or chromosomal loci in some types of glaucoma. ${ }^{10-12}$ In PDS-affected families, significant linkage has been observed between the disease phenotype and markers located on several chromosomal loci. GPDS1 (OMIM ID 600510) was the first genetic locus on chromosome 7q35-q36 found to be associated with PDS. ${ }^{13}$

Although the GPDS1 locus may include a gene associated with NTG, an association between the GPDS1 locus and NTG had not been previously investigated. The purpose of this study is to determine whether the GPDS1 locus is one of the candidate loci for developing NTG. We explored this potential in an association study using 20 microsatellite markers around the GPDS1 locus.

\section{Materials and methods}

\section{Participants}

We recruited 242 Japanese participants from the Yokohama City University, Yamanashi University, Gifu University, Kobe University, Yamaguchi University, Kumamoto University, Hokkaido University, Tokyo University, Niigata University, Kanazawa University, Hiroshima University, Tajimi Municipal Hospital, and Tokai University in Japan. Of these participants, 141 were diagnosed with NTG, and 101 served as controls. The criteria applied for the diagnosis of NTG were those proposed in our previous study. ${ }^{14}$ The mean patient age was $49.2 \pm 9.6$ years, and the male:female ratio was 0.87 . The mean refraction value was $-3.75 \pm 3.02$ diopters (D), and the mean deviation observed in the Humphrey ${ }^{\circledR}$ static visual field determination (Carl Zeiss Meditec, Oberkochen, Germany) was $-10.13 \pm 8.04 \mathrm{~dB}$. The control participants were of the same age and sex as the NTG patients, and they were not affected by glaucoma or any local or systemic illnesses that could result in optic disc or visual field changes. Furthermore, the control cases either had no myopia or had mild myopia with refractive errors of $-3.00 \mathrm{D}$ or less. All patients and control participants were of Japanese ethnicity, with similar social backgrounds and residing in the same urban area. Informed consent was obtained from all participants, and the study was conducted in accordance with the Declaration of Helsinki and its subsequent revisions.

\section{Analysis of repeat polymorphisms for 12 microsatellite loci}

Genomic DNA was extracted using the QIAamp DNA Blood Mini Kit (Qiagen, Hilden, Germany) or the guanidine method. In this association study, we selected 20 microsatellite loci that are located around the GPDS1 region on chromosome 7q35-36.3 and that have been established as informative polymorphic genetic markers (Figure 1). The markers were determined based on the National Center for Biotechnology Information guidelines for fine mapping. A polymerase chain reaction (PCR) was performed in a reaction mixture with a total volume of $12.5 \mu 1$, containing PCR buffer, genomic DNA, $0.2 \mathrm{mM}$ dinucleotide triphosphates (dNTPs), $0.5 \mu \mathrm{M}$ primers, and $0.35 \mathrm{U}$ Taq polymerase. The PCR conditions were as follows: $94^{\circ} \mathrm{C}$ for $5 \mathrm{~min}$, followed by 30 cycles of denaturation at $94^{\circ} \mathrm{C}$ for $30 \mathrm{sec}$, annealing at $56^{\circ} \mathrm{C}$ for $30 \mathrm{sec}$, extension at $72{ }^{\circ} \mathrm{C}$ for $1 \mathrm{~min}$, and a final elongation step at $72{ }^{\circ} \mathrm{C}$ for $10 \mathrm{~min}$. The reaction was carried out in a PCR thermal cycler (GeneAmp System 9700, Applied Biosystems, Foster City, CA, USA). The forward primer was labeled with 6-FAM (Sigma-Aldrich, St. Louis, USA) at the $5^{\prime}$ end (Table 1). To determine the number of microsatellite repeats, the PCR products were denatured at $97{ }^{\circ} \mathrm{C}$ for $2 \mathrm{~min}$, mixed with formamide, and electrophoresed in an ABI3130 Genetic Analyzer (Applied Biosystems). The number of microsatellite repeats was estimated automatically using GeneScan 672 software (Applied Biosystems) and the local Southern method with a size marker of GS500 TAMRA (Applied Biosystems).

\section{Statistical analysis}

Allele frequencies were estimated by direct counting. The significance of allele frequencies between the patient and control groups was evaluated by the Fisher's exact test. The probability of association was corrected by the Bonferroni inequality method, ie, by multiplying the obtained $\mathrm{P}$ values with the number of alleles compared. A Pc value of $<0.05$ was considered statistically significant. Statistical analyses were performed on a computer using SPSS software (version 10.1; SPSS Inc., Chicago, IL, USA). 


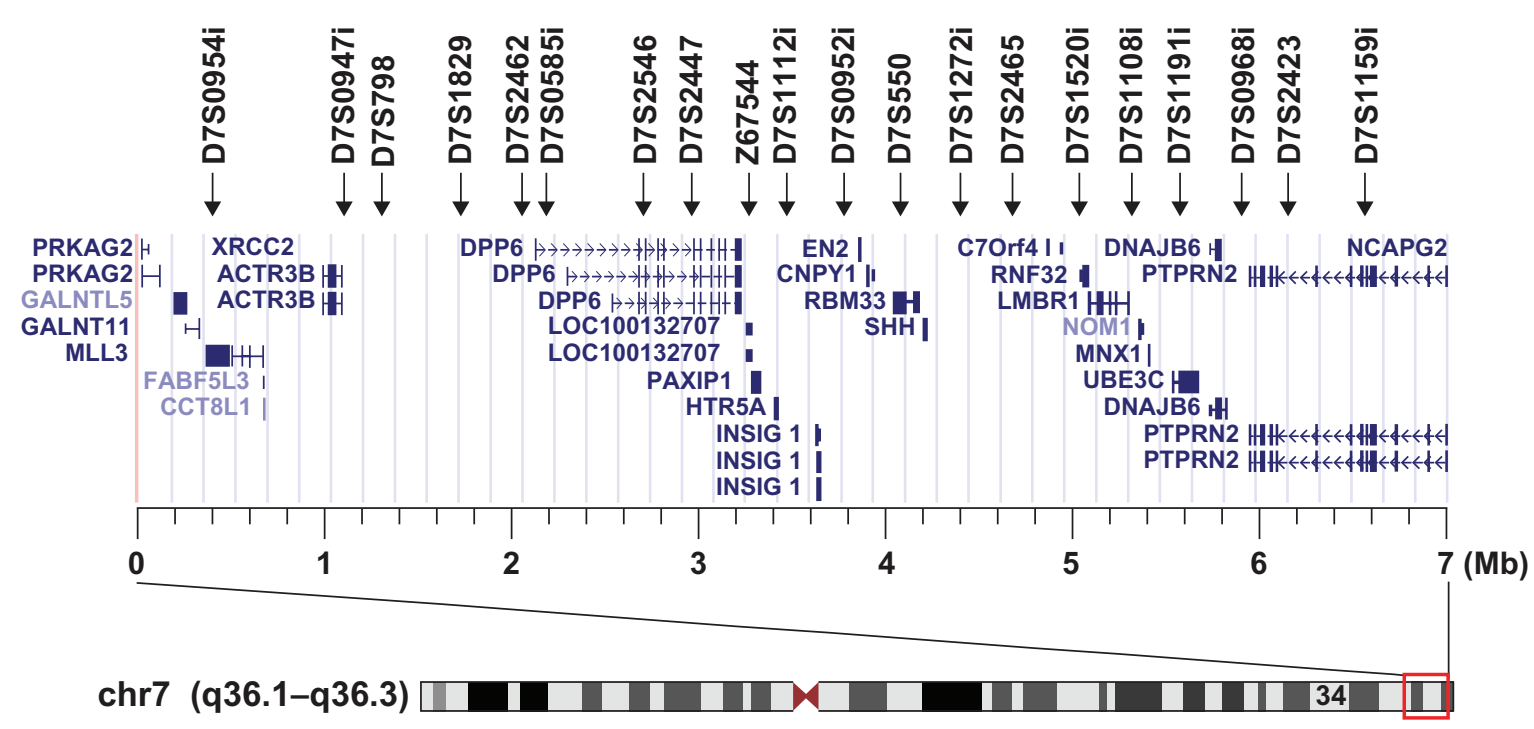

Figure I Location of 20 microsatellite markers used in this study and the genes around the GPDSI locus. Display of genes and chromosome seven results in the UCSC genome browser on Human Mar. 2006 Assembly at chr7: 151, 120,000-1 58, 120,000.

\section{Results}

We analyzed 20 microsatellite markers localized around GPDS1 for all patients and control participants (Figure 1). All allele frequencies were found to be in Hardy-Weinberg equilibrium proportion. Table 2 lists the allele frequencies of microsatellite markers in patients and control participants, with each allele designated by the size of its amplification. The 132 allele of D7S2462 was significantly associated with NTG, and the allele frequency was decreased in NTG cases as compared to controls ( $14.5 \%$ vs $26.2 \%, \mathrm{P}=0.0013$, $\mathrm{Pc}=0.019, \mathrm{OR}=0.48,95 \% \mathrm{CI}=0.30-0.75)$. Another two markers, D7S2465 and D7S2423, were also negatively associated with the risk of NTG. On the other hand, four markers - D7S2546, Z67544, D7S1272i, and D7S1108i were positively associated with the risk of NTG. However, these two negative associations and four positive associations did not retain statistical significance after Bonferroni correction $(\mathrm{P}<0.05, \mathrm{Pc}>0.05)$.

\section{Discussion}

Criteria for PDS were two of the following: midperipheral, radial iris transillumination defects; Krukenberg spindle; heavy trabecular meshwork pigmentation; and wide-open angle. ${ }^{9}$ Criteria for PG were PDS and two or more of three findings: IOP greater than $21 \mathrm{mmHg}$, optic nerve damage, or visual field loss. The probability of converting to pigmentary glaucoma was $10 \%$ at 5 years and $15 \%$ at 15 years. Young, myopic men were most likely to have pigmentary glaucoma. The most significant risk factor for conversion to pigmentary glaucoma was an IOP greater than $21 \mathrm{mmHg}$ at initial examination, whereas age, refractive error, and family history of glaucoma were not correlated with conversion. ${ }^{15}$

NTG is a critical and major subtype of POAG and shares many of the same pathologic characteristics with HTG except for elevated IOP. Notably, many other diseases cause atrophy of the optic nerve similar to that caused by NTG. Since the coexistence of these different diseases decreases statistical validation in the case-control analysis, we carefully selected the NTG patients using highly stringent criteria to select and distinguish the NTG phenotype from the other diseases that cause optic nerve atrophy.

The GPDS1 locus is responsible for PDS..$^{13}$ An association between the locus and NTG, however, had not been previously investigated. Therefore, in this study, we sequenced the repeat polymorphisms of 20 microsatellite loci within and around the GPDS1 locus to determine whether these repeat polymorphisms are associated with NTG in a Japanese population.

The 20 microsatellite markers selected for this study were located almost $100-700 \mathrm{~kb}$ apart from each other on chromosome 7q35-36.3; they were chosen to achieve dense coverage of the regions located around GPDS1 and to detect susceptible loci related to NTG. As a result, a relatively increased allele frequency was observed in the patient group for the four microsatellite markers D7S2546, Z67544, D7S1272i, and D7S1108i. Another three markers - D7S2462, D7S2465, and D7S2423 - were associated with lower risk of NTG. Of these markers, only D7S2462 remained 
Table I Primers used in polymerase chain reaction for amplification of microsatellite markers in this study

\begin{tabular}{|c|c|c|}
\hline Location & Orientation $^{a}$ & Sequence \\
\hline \multirow[t]{2}{*}{ D7S0954i } & $\mathrm{F}(\mathrm{NED})$ & 5'TTACATAAGGTACTTTCTGGTCAT3' \\
\hline & $\mathrm{R}$ & 5'TGGTCTCCAGCTACATCAG3' \\
\hline \multirow[t]{2}{*}{ D7S0947i } & $F(N E D)$ & 5'CTACTTTCCTGGTGTCTAGCTT3' \\
\hline & $\mathrm{R}$ & 5'GATAACAGCTGATAGGTAGATCAG3' \\
\hline \multirow[t]{2}{*}{ D7S798 } & $\mathrm{F}(\mathrm{VIC})$ & 5'AGCTGCAAAATAGTGGAAGTAG3' \\
\hline & $\mathrm{R}$ & 5'CATCAATTCACATAATGACCG3' \\
\hline \multirow[t]{2}{*}{ D7SI829 } & $\mathrm{F}(\mathrm{VIC})$ & 5'GGGGGTTTCAGAAGAGATGT3' \\
\hline & $\mathrm{R}$ & 5'CCTCCAAAATTTCTGAGCAA3' \\
\hline \multirow[t]{2}{*}{ D7S2462 } & $\mathrm{F}(\mathrm{FAM})$ & 5'GCCAAGATTGCACCACTAC $3^{\prime}$ \\
\hline & $\mathrm{R}$ & 5'TGTTAAAAGTTCCCCAAGC3' \\
\hline \multirow[t]{2}{*}{ D7S0585i } & $F(N E D)$ & 5'AATGAGCATTCTCAGTTTGAATAC3' \\
\hline & $\mathrm{R}$ & 5'ATCTGGTGAACGAGTAAATAACAC3' \\
\hline \multirow[t]{2}{*}{ D7S2546 } & $\mathrm{F}(\mathrm{FAM})$ & 5'GGAGGTTGAACAACTCTGAATAC3' \\
\hline & $\mathrm{R}$ & 5'CACGCCAGGGTCTATCTT3' \\
\hline \multirow[t]{2}{*}{ D7S2447 } & $\mathrm{F}(\mathrm{NED})$ & 5'GTCAGATGTAGGAACCAAGC3' \\
\hline & $\mathrm{R}$ & 5'TCTCACTGTACTCAGCCTGT3' \\
\hline \multirow[t]{2}{*}{ Z67544 } & $\mathrm{F}(\mathrm{VIC})$ & 5'CAACTTAAAATACGCTGTTACC $3^{\prime}$ \\
\hline & $\mathrm{R}$ & 5'GCTTTTTCAGACCAAATAACTTAC3' \\
\hline \multirow[t]{2}{*}{ D7SIIII2i } & $F(N E D)$ & 5'GAGAATCTAATGAAAGCTGTGG3' \\
\hline & $\mathrm{R}$ & 5'TTCAGGGCATCCAAGAAG3' \\
\hline \multirow[t]{2}{*}{ D7S0952i } & $\mathrm{F}(\mathrm{NED})$ & 5'AGATATTTGGCTAAACATGGTC3' \\
\hline & $\mathrm{R}$ & 5'TCTTCAGAGATACAGAAGCAATAG3' \\
\hline \multirow[t]{2}{*}{ D7S550 } & $\mathrm{F}(\mathrm{FAM})$ & 5'TCTCATCTGTGAATGCACTATC3' \\
\hline & $\mathrm{R}$ & 5'GCAGTTGGGTTATTTCAAGTC3' \\
\hline \multirow[t]{2}{*}{ D7SI272i } & $\mathrm{F}(\mathrm{PET})$ & 5'AATGTCCTGGAGGCTGAG3' \\
\hline & $\mathrm{R}$ & 5'AAAGACTTGGTGAGACCTTCTC3' \\
\hline \multirow[t]{2}{*}{ D7S2465 } & $\mathrm{F}(\mathrm{PET})$ & 5'ACCTGGGCAACAGAGTGAG3' \\
\hline & $\mathrm{R}$ & 5'CTTCAAAGAGTTTATGCTTATGTGG3' \\
\hline \multirow[t]{2}{*}{ D7SI520i } & $\mathrm{F}(\mathrm{PET})$ & 5'GCTGTTAGCATCTGGTTAATTTAC3' \\
\hline & $\mathrm{R}$ & 5'AAACTGAAGTCTGCCATCTATTAG3' \\
\hline \multirow[t]{2}{*}{ D7SII08i } & $\mathrm{F}(\mathrm{FAM})$ & 5'ACTTACTTATCCTACTGATCCGTG3' \\
\hline & $\mathrm{R}$ & 5'TGAACCATAAATTACCTCCATTC3' \\
\hline \multirow[t]{2}{*}{ D7SII9Ii } & $\mathrm{F}(\mathrm{VIC})$ & 5'CACTCTATTTGCATGGTGAAC3' \\
\hline & $\mathrm{R}$ & 5'TCCAGTAGAGGGAGCTCAG3' \\
\hline \multirow[t]{2}{*}{ D7S0968i } & $F(N E D)$ & 5'CACATCCTTTATACCTACATTCAG3' \\
\hline & $\mathrm{R}$ & 5'TAATTTATCTAGAAGGTCAGCAAA3' \\
\hline \multirow[t]{2}{*}{ D7S2423 } & $\mathrm{F}(\mathrm{NED})$ & 5'CTTCAGACCTTCAGTTGATGAT3' \\
\hline & $\mathrm{R}$ & 5'GCTCTCAGACACATTTTCCA3' \\
\hline \multirow[t]{2}{*}{ D7SII59i } & $\mathrm{F}(\mathrm{VIC})$ & 5'CTTGGAGTCAAAGAGGCC3' \\
\hline & $\mathrm{R}$ & 5'CTTTCCTGACTCCTTGATTAGAG3' \\
\hline
\end{tabular}

Note: ${ }^{a}$ Dye label.

significantly associated with NTG after Bonferroni correction (Pc $<0.05)$. Therefore, there is one statistically appreciable difference between the NTG group and the control group in these 20 microsatellite markers located over a 6-Mb genomic region around GPDS1.
The dipeptidyl-peptidase 6 (DPP6) gene is the nearest gene to the D7S2462 marker. DPP6 is a type II membrane protein that binds voltage-gated neuronal potassium channels (A-type) and alters their expression and biophysical properties. ${ }^{16}$ Recently, genetic variants in DPP6 have been 
Table 2 Frequencies of 20 microsatellite markers in normal-tension glaucoma (NTG) patients and healthy participants

\begin{tabular}{|c|c|c|c|c|c|c|c|}
\hline \multirow[t]{2}{*}{ Marker } & \multirow{2}{*}{$\begin{array}{l}\text { Occurrence } \\
\text { of allele }\end{array}$} & \multirow[t]{2}{*}{ Allele $^{a}$} & \multicolumn{2}{|l|}{ Frequencies (\%) } & \multirow[t]{2}{*}{$\mathbf{P}$} & \multirow[t]{2}{*}{ Pc } & \multirow{2}{*}{$\begin{array}{l}\text { Odds ratio } \\
(95 \% \mathrm{Cl})\end{array}$} \\
\hline & & & $\begin{array}{l}\text { NTG patients } \\
(2 n=282)\end{array}$ & $\begin{array}{l}\text { Healthy } \\
\text { participants } \\
(2 n=202)\end{array}$ & & & \\
\hline D7S0954i & 9 & 310 & 3.5 & 2.0 & 0.31 & & \\
\hline D7S0947i & 7 & 151 & 3.2 & 2.5 & 0.64 & & \\
\hline D7S798 & 8 & 211 & 7.8 & 4.0 & 0.08 & & \\
\hline D7SI 829 & 13 & 391 & 6.0 & 4.0 & 0.31 & & \\
\hline D7S2462 & 15 & 132 & 14.5 & 26.2 & 0.0013 & 0.019 & 0.48 \\
\hline D7S0585i & 9 & 324 & 4.3 & 3.0 & 0.46 & & \\
\hline D7S2546 & 8 & 239 & 13.8 & 6.4 & 0.0096 & 0.077 & 2.33 \\
\hline D7S2447 & 4 & 175 & 53.5 & 52.5 & 0.82 & & \\
\hline Z67544 & 6 & $15 \mid$ & 6.4 & 1.5 & 0.0091 & 0.055 & 3.99 \\
\hline D7SIII2i & 6 & 121 & 44.7 & 42.1 & 0.57 & & \\
\hline D7S0952i & 3 & 450 & 1.2 & 0.5 & 0.21 & & \\
\hline D7S550 & 13 & 192 & 1.2 & 0.5 & 0.21 & & \\
\hline$D 7 S I 272 i$ & 6 & 156 & 20.2 & 13.4 & 0.0499 & 0.30 & 1.64 \\
\hline D7S2465 & 14 & 175 & 22.7 & 32.2 & 0.020 & 0.28 & 0.62 \\
\hline D7SI520i & 8 & 300 & 3.2 & 3.0 & 0.58 & & \\
\hline D7SII08i & 14 & 188 & 3.2 & 0.5 & 0.040 & 0.56 & 6.63 \\
\hline D7SII9li & 6 & 181 & 0.7 & 0.5 & 0.77 & & \\
\hline D7S0968i & 6 & 357 & 50.0 & 44.6 & 0.24 & & \\
\hline D7S2423 & 5 & 250 & 19.9 & 29.2 & 0.017 & 0.086 & 0.60 \\
\hline D7SII59i & 8 & 115 & 13.8 & 9.4 & 0.14 & & \\
\hline
\end{tabular}

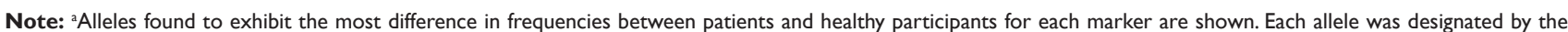
size of its amplification.

reported to show a significant association with human neural diseases such as amyotrophic lateral sclerosis and autism. ${ }^{17-21}$ Since potassium channels are considered to play an important role in protecting against ischemia and the development of apoptosis, ${ }^{22}$ DPP6 variants may act as risk factors in the development of NTG.

In conclusion, we performed an association analysis of the GPDS1 locus using microsatellite markers in NTG patients and found that there was an NTG-associated region in the GPDS1 locus. Therefore, further association studies using single nucleotide polymorphisms in the resistant region might help to identify the pathogenic gene(s) related to NTG.

\section{Acknowledgments}

This study was supported by grants-in-aid from the Ministry of Education, Science, Sports, and Culture of Japan; a grant from the Ministry of Health, Labour, and Welfare, Japan; and a grant from the Johnson and Johnson KK Vision Care Company. The authors report no conflicts of interest in this work.

\section{References}

1. Foster A, Resnikoff S. The impact of Vision 2020 on global blindness. Eye. 2005;19(10):1133-1135.

2. Quigley HA. Number of people with glaucoma worldwide. $\mathrm{Br} J$ Ophthalmol. 1996;80(5):389-393.

3. Bonomi L, Marchini G, Marraffa M, et al. Prevalence of glaucoma and intraocular pressure distribution in a defined population. The EgnaNeumarkt Study. Ophthalmology. 1998;105:209-215.

4. Iwase A, Suzuki Y, Araie M, et al; For the Tajimi Study Group, Japan Glaucoma Society. The prevalence of primary open-angle glaucoma in Japanese: the Tajimi Study. Ophthalmology. 2004;111:1641-1648.

5. Broadway DC, Drance SM. Glaucoma and vasospasm. Br J Ophthalmol. 1998;82:862-870.

6. Fontana L, Poinoosawmy D, Bunce CV, et al. Pulsatile ocular blood flow investigation in asymmetric normal tension glaucoma and normal subjects. Br J Ophthalmol. 1998;82:731-736.

7. Ishida K, Yamamoto T, Kitazawa Y. Clinical factors associated with progression of normal-tension glaucoma. J Glaucoma. 1998;7: 372-377.

8. Drance S, Anderson DR, Schulzer M; for the Collaborative NormalTension Glaucoma Study Group. Risk factors for progression of visual field abnormalities in normal-tension glaucoma. Am J Ophthalmol. 2001;131:699-708.

9. Kollu Nageswara Rao, Robert Ritch Syril K, Dorairaj, et al. Exfoliation syndrome and exfoliation glaucoma-associated LOXL1 variations are not involved in pigment dispersion syndrome and pigmentary glaucoma. Mol Vis. 2008;14:1254-1262. 
10. Fan BJ, Wang DY, Lam DS, Pang CP. Gene mapping for primary open angle glaucoma. Clin Biochem. 2006;39(3):249-258.

11. Wiggs JL. Genetic etiologies of glaucoma. Arch Ophthalmol. 2007; 125(1):30-37.

12. Vasiliou V, Gonzalez FJ. Role of CYP1B1 in glaucoma. Аnпи Rev Pharmacol Toxicol. 2008;48:333-358.

13. Andersen JS, Pralea AM, DeiBono EA, et al. A gene responsible for the pigment dispersion syndrome maps to chromosome 7q35-q36. Arch Ophthal. 1997;115(3):384-388.

14. Shibuya E, Meguro A, Ota M, et al. Association of Toll-like receptor 4 gene polymorphisms with normal tension glaucoma. Invest Ophthalmol Vis Sci. 2008;49(10):4453-4457.

15. Yasmin Siddiqui, Richard d, Ten Huizen J, Douglas Cameron, et al. What is the risk of developing pigmentary glaucoma from pigment dispersion syndrome? Am J Ophthalmol. 2003;135(6):794-799.

16. Nadal MS, Ozaita A, Amarillo Y, et al. The CD26-related dipeptidyl aminopeptidase-like protein DPPX is a critical component of neuronal A-type K+ channels. Neuron. 2003;37(3):449-461.
17. van Es MA, van Vught PW, Blauw HM, et al. Genetic variation in DPP6 is associated with susceptibility to amyotrophic lateral sclerosis. Nat Genet. 2008;40(1):29-31.

18. Cronin S, Berger S, Ding J, et al. A genome-wide association study of sporadic ALS in a homogenous Irish population. Hum Mol Genet. 2008;17(5):768-774.

19. Garber K. Genetics. The elusive ALS genes. Science. 2008; 319(5859):20

20. Del Bo R, Ghezzi S, Corti S, et al. DPP6 gene variability confers increased risk of developing sporadic amyotrophic lateral sclerosis in Italian patients. J Neurol Neurosurg Psychiatry. 2008;79(9):1085.

21. Marshall CR, Noor A, Vincent JB, et al. Structural variation of chromosomes in autism spectrum disorder. Am J Hum Genet. 2008;82(2): 477-488.

22. Burg ED, Remillard CV, Yuan JXJ. $\mathrm{K}^{+}$channels in apoptosis. J Membr Biol. 2006;209:3-20. 